\title{
Das Kind X: Verfassungsrechtliche Fragen der anonymen Kindesabgabe*
}

\section{Einführung}

„Kinder sind das köstlichste Gut eines Volkes,“verkündet Art. 125 Abs. 1 S. 1 BV, ohne Zweifel einer der schönsten Sätze der bayerischen Verfassung. Die besondere Affinität Bayerns zum Barock wird hier gewissermaßen in Rechtsworte gegossen. Die meisten Kinder benötigen diese prosaische Fürsprache durch das Verfassungsrecht zum Glück nicht. Sie sind das köstlichste Gut ihrer Eltern. Diese Beziehung beruht auf sittlichen Standards, nicht auf Rechtsnormen, und seien sie auch noch so wohlklingend. Einvernehmliche elterliche Liebe, die dem Wohle des Kindes dient, benötigt keine rechtliche Regulierung. ${ }^{1}$ Erst wenn soziale Konventionen nicht mehr greifen, bestimmen Rechtsnormen und entscheiden außerfamiliäre Institutionen, was dem Kindeswohl dienlich ist.

Manches Kind benötigt den Schutz des Rechts schon vor oder kurz nach seiner Geburt. Dabei hat sich die Einsicht durchgesetzt, dass wirksamer Schutz in dieser Lebensphase des Kindes nur mit und nicht gegen die Mutter verwirklicht werden kann und daher Beratung und soziale Hilfsangebote der beste Lebensschutz sind. ${ }^{2}$ Doch nicht jedes Hilfsangebot hält, was es verspricht. Besonders kontrovers wird derzeit über Einrichtungen diskutiert, in denen neugeborene Kinder anonym abgegeben/geboren werden können. Sie sollen verhindern, dass Mütter in extremen psychosozialen Lebenssituationen ihre Neugeborenen aussetzen oder töten. Im Wesentlichen kann man zwischen zwei Arten anonymer Kindesabgaben unterscheiden: ${ }^{3}$

Bei den überwiegend von katholischen Krankenhäusern und privaten Vereinen betriebenen Babyklappen wird eine Öffnung in einer Hauswand eingerichtet, hinter der sich ein Wärmebett befindet, in das das Neugeborene eingelegt werden kann. Unmittelbar danach schließt sich die Klappe; der Mutter wird ein gewisser Zeitraum eingeräumt sich unerkannt zu entfernen, bevor im Haus ein Signal ertönt, das das medizinische Personal auf die Benutzung der Babyklappe aufmerksam macht. Das Angebot wird mit Informationen über Beratungsmöglichkeiten und oftmals auch mit Verfahrensvorschlägen verbunden, die der späteren Identifikation für den Fall dienen, dass die Mutter

* Der nachfolgende Text beruht auf einer Stellungnahme im Rahmen einer Anhörung beim Deutschen Ethikrat am 23. 10. 2008; vgl. das Wortprotokoll der Anhörung unter www.ethikrat.org/der_files/Wortprotokoll_2008-10-23_Website.pdf - Der Autor ist Inhaber des Lehrstuhls für Öffentliches Recht, Sozialrecht und Gesundheitsrecht und Leiter der Forschungsstelle für Medizin- und Gesundheitsrecht an der Juristischen Fakultät der Universität Regensburg. Er dankt Frau Susanne Henck für ihre engagierte Mitarbeit.

1 H. Huba, Recht und Liebe, FamRZ 1989, 127 (128); G. Roellecke, Kinder, Kinder, NJW 1994, 1263 (1263f.).

2 Schwangeren- und Familienhilfegesetz (SFHG) v. 27. 7. 1992 (BGBl I, 1398) sowie das wegen BVerfGE 88, 203 notwendig gewordene Änderungsgesetz v. 21. 8. 1995 (BGBl I, 1050).

3 Vgl. zum Folgenden C. Mielitz, Anonyme Kindesabgabe, 2006, S. 20ff., die noch das eher selten praktizierte Übergabesystem als dritte Form der anonymen Kindesabgabe behandelt (S. 21). 
das Kind zurückbekommen möchte. Eine Umfrage der Bundesregierung bei den Ländern registrierte im Sommer 200776 Babyklappen. ${ }^{4}$

Anonyme Geburten unterscheiden sich von der Babyklappe in mehrfacher Hinsicht. In Notstandssituationen werden sie selbstverständlich schon seit jeher praktiziert, weil Ärzte und Hebammen strafrechtlich ( $\$ 323$ c StGB) verpflichtet sind, bei bevorstehenden Entbindungen auch dann Hilfe zu leisten, wenn die werdende Mutter ihre Identität nicht preisgeben möchte. Bei den hier allein zu diskutierenden Angeboten anonymer Geburten erfolgt die ärztlich begleitete Entbindung hingegen mit der Garantie, dass die Entbindende ihre Identität nicht preisgeben muss und der Anbieter die Kosten der Geburt trägt. Das Angebot wird meist mit einer auf Preisgabe der Identität zielenden Beratung verbunden. Die Mutter kann die Einrichtung nach der Entbindung mit oder ohne ihr Neugeborenes wieder verlassen. Als einziges Land lässt Frankreich Angebote der anonymen Geburten bereits seit 1941 zu. ${ }^{5}$ Valides Datenmaterial zu den Angeboten der anonymen Geburt gibt es nicht. ${ }^{6}$

Einrichtungen, die anonyme Kindesabgaben anbieten, sind nicht Bestandteil des staatlichen Schutzkonzepts für das ungeborene und neugeborene Leben, ja sie verstoßen, wie im Folgenden zunächst kurz zu skizzieren ist, gegen geltendes Recht (B.). Schwerpunkt des Beitrages ist daher die Frage, ob eine Änderung dieser Rechtsnormen und damit eine Legalisierung der anonymen Kindesabgabe verfassungsrechtlich zulässig wäre (dazu C.-E.).

\section{Die Rechtswidrigkeit der anonymen Kindesabgabe de lege lata}

\section{Personenstandsrechtliche Anzeigepflichten}

Die Geburt eines Kindes muss dem zuständigen Standesamt binnen einer Woche angezeigt werden, § 18 S. 1 PStG. Das ist von außerordentlicher Bedeutung, weil die Aufnahme der Anzeige die Entstehung der familienrechtlichen Beziehungen dokumentiert und zudem die zuständigen staatlichen Stellen (Jugendamt, Vormundschaftsgericht) ihre Verantwortung gegenüber dem Kind nur ausüben können, wenn sie von seiner Existenz erfahren. Zur Anzeige verpflichtet ist nach § 19 S. 1 Nr. 1 PStG jeder Elternteil des Kindes, wenn er sorgeberechtigt ist. Wenn die sorgeberechtigten Eltern an der Anzeige gehindert sind, ist auch jede andere Person zur Anzeige verpflichtet, die bei der Geburt zugegen war oder von der Geburt aus eigenem Wissen unterrichtet ist (§ 19 S. 2 i.V.m. S. 1 Nr. 2 PStG). Bei Geburten in Krankenhäusern und sonstigen Einrichtungen, in denen Geburtshilfe geleistet wird, ist nach $\S 20$ S. 1 PStG der Träger der Einrichtung zur Anzeige verpflichtet. Davon bleiben aber die Anzeigepflicht der Eltern und ihre Aus-

4 BT-Drucks. 16/7220, S. 26-28. Eine unvollständige Erhebung geht von insgesamt 143 seit 2001 abgegebenen Kindern aus, davon allein $25 \%$ in Berlin! Die genaue Zahl der abgegebenen Kinder lässt sich nicht feststellen, da Nordrhein-Westfalen die Frage nicht beantwortet und Bayern keine Erkenntnisse hat; nach Auskunft von U. Riedel, Anonyme Geburt, in: Deutscher Ethikrat. Wortprotokoll über den öffentlichen Teil der Sitzung vom 26. 6. 2008, S. 3, handelt es sich um weitere 34 Babyklappen.

5 Dazu Mielitz (Fn. 3), S. 33ff.

6 Vgl. die nur unvollständigen Angaben in BT-Drucks. 16/7220, S. 21-23. 
kunftspflicht zu Angaben, die die Einrichtung nicht machen kann, unberührt, § 20 S. 3 PStG. Diese am 1. 1. 2009 in Kraft getretene Neuregelung des Personenstandsrechts hat erhebliche Auswirkungen auch auf die Anzeige- und Auskunftspflichten bei anonymen Geburten:

- Nach $\S 17$ Abs. 1 Nr. 5 PStG a. F. war die Mutter bei einer Geburt im Krankenhaus nur nachrangig nach dem Leiter der Einrichtung zur Anzeige verpflichtet. Nunmehr bestehen beide Anzeigepflichten nebeneinander. Auch unterscheidet $\S 20$ S. 3 PStG nunmehr zwischen einer Anzeige- und einer darüber hinaus gehenden Auskunftspflicht. Personenstandsrechtlich ist daher insbesondere die Mutter verpflichtet, auch den Namen des Vaters anzuzeigen. ${ }^{7}$

- Aus der gesetzlichen Differenzierung zwischen Anzeige- und Auskunftspflicht folgt, dass die Träger von Einrichtungen, die anonyme Geburten anbieten, nur zur Anzeige, nicht aber zur Auskunft verpflichtet sind. ${ }^{8}$ Der Betreiber einer Babyklappe muss die Geburt des Kindes nur als Findelkind bei der Gemeindebehörde anzeigen, § 24 Abs. 1 S. 1 PStG. ${ }^{9}$ Die Anbieter handeln daher nicht personenstandsrechtswidrig, wenn sie die Geburt des Kindes anzeigen und dabei nur diejenigen Daten mitteilen, die sie selbst kennen.

Wer auf Grund des Personenstandsgesetzes zu Anzeigen verpflichtet ist, kann hierzu vom Standesamt durch Festsetzung eines Zwangsgeldes angehalten werden. Die Verletzung dieser Pflichten kann nach $\S 70$ Abs. 1 Nr. 1-3 PStG zudem ein Bußgeld zur Folge haben. Jedenfalls die Mutter kann sich ferner wegen Personenstandsunterdrückung ( $\$ 169$ Var. 3 StGB) und Verletzung der Unterhaltspflicht nach $§ 170$ StGB strafbar machen. ${ }^{10}$ Die Strafbarkeit der Anbieter ist zwar umstritten, ${ }^{11}$ jedenfalls begünstigen sie mit ihrem Angebot die Rechtsverstöße der abgebenden Mutter; abgesehen davon, dass sie offenbar auch ihre eigenen personenstandsrechtlichen Verpflichtungen teilweise nicht einhalten. ${ }^{12}$ Die Meldung erfolgt oftmals erst im Rahmen des Adoptionsverfahrens, um den Müttern eine Frist zum Überdenken ihrer Entscheidung einzuräumen. ${ }^{13}$

\section{2. Übernahme der Vormundschaft}

Uneinheitlich wird die Übernahme der Vormundschaft gehandhabt. Das Kind erhält, da seine Eltern nicht bekannt sind, gemäß § 1773 Abs. 2 BGB einen Vormund, den das Vormundschaftsgericht nach Anhörung des Jugendamtes auswählt (§1779 Abs. 1

7 So zutreffend bereits zum alten Recht $D$. Elbel, Rechtliche Bewertung anonymer Geburt und Kindesabgabe, 2007, S. 53.

8 Elbel (Fn. 7), S. 44ff; anders noch S. Benöhr/I. A. Muth, „Babyklappe“ und ,anonyme Geburt“ - Im Widerstreit zwischen Hilfeleistung und Gesetzesverstoß, KJ 2001, 405 (415).

9 F. Bardenberg, Das Recht des Kindes auf Kenntnis der eigenen Abstammung unter Berücksichtigung der Problematik der anonymen Geburt, 2006, S. 99.

10 S. Neuheuser, Begründet die Weggabe eines Neugeborenen in eine Babyklappe den Anfangsverdacht einer Straftat?, NStZ 2001, 175 (177).

11 Elbel (Fn. 7), S. 59ff. und Mielitz (Fn. 3), S. 114ff.

12 C. Swientek, Die Wiederentdeckung der Schande - Babyklappen und anonyme Geburt, 2001, S. 13.

13 Mielitz (Fn. 3), S. 22 und Riedel (Fn. 4), S. 5. 
BGB) und nach Maßgabe von $\S 1789$ BGB bestellt. Dabei fällt die Wahl teilweise auf das Jugendamt ( $\$ 1791$ b BGB), teilweise aber auch auf Mitarbeiter der Einrichtung der anonymen Kindesabgabe ( $\$ 1791$ a BGB), was als problematisch gilt, weil der Anbieter der Mutter einerseits Anonymität zugesichert hat, andererseits aber als Vormund im Interesse des Kindes dazu verpflichtet ist, dessen leibliche Eltern ausfindig zu machen. ${ }^{14}$ Jugendamt bzw. Einrichtung vermitteln das Kind sodann in eine Pflegefamilie, die ggfs. auch schon für die Adoption in Frage kommt.

\section{Adoption des Kindes}

Die Adoption des anonym abgegebenen Kindes setzt neben dessen Einverständnis (regelmäßig nach § 1746 Abs. 2 BGB also seines Vormundes) nach § 1747 BGB das der Eltern voraus. Diese können ihre Einwilligung nach $\S 1747$ Abs. 2 S. 1 BGB erst acht Wochen nach der Geburt erteilen und müssen dies nach $§ 1750$ BGB gegenüber dem Vormundschaftsgericht erklären. Selbst wenn man die anonyme Kindesabgabe als eine solche Erklärung deuten wollte, wäre diese aufgrund der Nichteinhaltung der genannten Bestimmungen unerheblich. Die Einwilligung ist zwar entweder nach § 1747 Abs. 4 BGB bei dauerhaft unbekanntem Aufenthalt der Eltern verzicht- oder nach $\S 1748$ Abs. 2 BGB ersetzbar. ${ }^{15}$ Vor einem Verzicht muss das Vormundschaftsgericht nach $\S 12$ FGG aber wenigstens 6 Monate versuchen, die Identität der Eltern zu ermitteln und eine eventuelle Ersetzung darf nach $\S 1748$ Abs. 2 S. 3 BGB frühestens fünf Monate nach der Geburt des Kindes erfolgen. In der Praxis erfolgt sie wegen $§ 1744$ BGB (angemessene Probezeit) oftmals erst später. Diese Fristen werden aber bei anonym abgegebenen Kindern offenbar regelmäßig nicht eingehalten, weil die Ermittlung der Identität der Eltern wegen der der Mutter zugesicherten Anonymität für sinnlos gehalten wird.

\section{Gesetzesinitativen}

Die anonyme Kindesabgabe verstößt insbesondere gegen personenstands- und strafrechtliche Bestimmungen. Sie bewegt sich damit nicht etwa in einer Grauzone, sondern ist schlicht rechtswidrig. ${ }^{16}$

Schon seit einiger Zeit besteht Einigkeit darüber, dass die Nichtdurchsetzung geltenden Rechts unbefriedigend ist. Daraus ist aber bislang der Schluss gezogen worden, dass das Recht dem Angebot und nicht das Angebot dem Recht anzupassen ist. Vier Geset-

14 Vgl. für diese Kontroverse die Entscheidung LG Flensburg v. 19.7.2002 - 5 T 250/02-5 T $255 / 02$.

15 Zum umstrittenen Verhältnis zwischen den beiden Vorschriften Elbel (Fn. 7), S. 93ff.

16 C. Katzenmeier, Rechtsfragen der „Babyklappe“ und der medizinisch assistierten ,,anonymen Geburt“, FamRZ 2005, 1134 /1135); R. Richardi, Lebensschutz durch Legalisierung der anonymen Geburt?, in: H.-J. Ahrens/C. v. Bar/G. Fischer/J. Taupitz/A. Spickhoff (Hrsg.), Festschrift für Erwin Deutsch zum 85. Geburtstag, 2009, i.E.; A. Wolf, Über Konsequenzen aus den gescheiterten Versuchen, Babyklappen und ,anonyme“ Geburten durch Gesetz zu legalisieren, FPR 2003, 112 (113). 
zinitiativen aus der Mitte des Bundestages ${ }^{17}$ und aus dem Bundesrat ${ }^{18}$ einte das gemeinsame Ziel, die anonyme Kindesabgabe unter bestimmten Voraussetzungen zu legalisieren. Alle sind wegen verfassungsrechtlicher Bedenken nicht weiter verfolgt worden, weil sie dem am meisten Betroffenen nicht gerecht geworden sind: dem Kind, das nicht weiß, wo es herkommt. Anonyme Kindesabgaben werden gleichwohl nach wie vor geduldet. Im Jahre 2007 hat die Bundesregierung die Daten aus den Ländern zusammengetragen und einen Regelungsbedarf vermerkt. ${ }^{19}$

\section{Die betroffenen Grundrechte}

Verfassungsrechtlich ist fraglich, ob der Staat durch die derzeit praktizierte Duldung der anonymen Kindesabgabe Grundrechte verletzt oder durch eine Legalisierung verletzen würde. Betroffen ist neben dem Kind auch der nicht abgebende Elternteil, wobei im Folgenden der Einfachheit halber auf den leiblichen Vater abgestellt wird.

\section{Art. 2 Abs. 1 i.V.m. Art. 1 Abs. 1 GG}

Das allgemeine Persönlichkeitsrecht (Art. 2 Abs. 1 i.V.m. Art. 1 Abs. 1 GG) schützt „die Möglichkeit, sich als Individuum nicht nur sozial, sondern auch genealogisch in eine Beziehung zu anderen zu setzen. “Es umfasst daher ,das Recht des Kindes auf Kenntnis der eigenen Abstammung ebenso wie es einem Mann das Recht auf Kenntnis einräumt, ob ein Kind von ihm abstammt. " ${ }^{20}$ Geschützt ist damit das Wissen über die Vor- und Nachfahren. ${ }^{21}$

\section{a) Schutz des Kindes}

Das Recht des Kindes auf Kenntnis seiner Abstammung leitet das Bundesverfassungsgericht aus dem Umstand ab, dass Verständnis und Entfaltung der Individualität mit der Kenntnis der für sie konstitutiven Faktoren eng verbunden sind. Abstammung sei mehr als eine nur biologische Kategorie: „Bei Individualitätsfindung und Selbstverständnis handelt es sich vielmehr um einen vielschichtigen Vorgang, in dem biologisch gesicherte Erkenntnisse keineswegs allein ausschlaggebend sind. Als Individualisierungsmerkmal gehört die Abstammung zur Persönlichkeit, und die Kenntnis der Herkunft bietet dem Einzelnen unabhängig vom Ausmaß wissenschaftlicher Ergebnisse wichtige Anknüpfungspunkte für das Verständnis und die Entfaltung der eigenen Individualität. "22 Mit dieser Begründung hat das Bundesverfassungsgericht gesetzliche Bestim-

17 Gesetzentwurf der CDU/CSU-Fraktion v. 12. 10. 2000, BT-Drucks. 14/4425 und interfraktioneller Gesetzentwurf (ohne PDS-Fraktion) v. 23. 4. 2002, BT-Drucks. 14/8856.

18 Gesetzesvorschläge des Landes Baden-Württemberg v. 6. 6. 2002, BR-Drucks. 506/02 und vom 24. 9. 2004, BR-Drucks. 682/04.

19 BT-Drucks. 16/7220.

20 BVerfGE 117, 202 (226).

21 Kritisch zu dieser Gleichstellung Elbel (Fn. 7), S. 161.

22 BVerfGE 79, 256 (268 f.); vgl. ferner BVerfGE 90, 263 (270 f.); 96, 56 (63). 
mungen, die einem Kind die gerichtliche Klärung der eigenen Abstammung gänzlich verwehrten ${ }^{23}$ oder nur innerhalb einer bestimmten Frist gewährten, ${ }^{24}$ für verfassungswidrig erklärt. Auch der Europäische Gerichtshof für Menschenrechte (EGMR) erkennt ein Grundrecht auf Kenntnis der eigenen Abstammung an, leitet dieses aber aus dem in Art. 8 EMRK gewährleisteten Recht auf Achtung des Privatlebens ab. Dieses werde, so der EGMR in der Entscheidung Odiévre/Frankreich, durch die in Frankreich zugelassene anonyme Geburt berührt. ${ }^{25}$

Gegen die Berührung des Schutzbereiches könnte die Aussage des Bundesverfassungsgerichts sprechen, dass Art. 2 Abs. 1 i.V.m. Art. 1 Abs. 1 GG kein Recht auf Verschaffung von Kenntnissen der eigenen Abstammung verleiht, sondern nur vor der Vorenthaltung erlangbarer Informationen schützt. ${ }^{26}$ Das Bundesverfassungsgericht begegnet damit aber nur dem möglichen Einwand, dass es Fälle gibt, ,in denen die Abstammung unaufklärbar bleibt und die Persönlichkeitsentfaltung ohne diese Kenntnis erfolgen muss. "27 In einem solchen Fall sind die Behörden tatsächlich nicht gehalten, das Unaufklärbare aufzuklären. Die Legalisierung der anonymen Kindesabgabe macht aber das grundsätzlich Aufklärbare unaufklärbar. Der Gesetzgeber würde einen aktiven Beitrag dazu leisten, das Kind von der „Hauptinformationsquelle zu seiner Vergangenheit und persönlichen Entwicklung " 28 und damit von grundsätzlich erlangbaren Informationen abzuschneiden. Dass später kein grundrechtlicher Anspruch mehr auf Verschaffung dieser Informationen besteht, belegt gerade die Irreversibilität einer Zulassung der anonymen Kindesabgabe.

\section{b) Schutz des leiblichen Vaters}

Das Wissen um die Abstammung des Kindes hat, so das Bundesverfassungsgericht im Hinblick auf heimliche Vaterschaftstests, auch ,maßgeblichen Einfluss auf das Selbstverständnis des Mannes sowie die Rolle und Haltung, die er dem Kind und der Mutter gegenüber einnimmt.“29

Der Vater ist zwar bei der anonymen Kindesabgabe anders betroffen als beim Verbot des heimlichen Vaterschaftstests: Während es dort um den Vater geht, der seine Vaterschaft hinterfragt, geht es hier um den Vater, der von seiner Vaterschaft entweder nichts weiß oder sie jedenfalls nicht praktizieren kann. In beiden Fällen möchte der Betroffene aber wissen, wer sein Nachfahre ist, um damit eine für die Persönlichkeitsbildung wichtige genealogische Beziehung zu begründen. Art. 1 Abs. 1 i. V. m. Art. 2 Abs. 1 GG schützt daher auch das Recht auf Kenntnis der eigenen Nachfahren. ${ }^{30}$

23 BVerfGE 79, 256.

24 BVerfGE 90, 263.

25 EGMR, NJW 2003, 2145 (2146 Nr. 29).

26 BVerfGE 79, 256 (269).

27 BVerfGE 79, 256 (269).

28 Formulierung von EGMR v. 7.7.1989, Serie A, Bd. 160, S. 15 Nr. 36.

29 BVerfGE 117, 202 (226).

30 J. Gernhuber/D. Coester-Waltjen, Familienrecht, 5. Aufl. 2006, § 52 Rn. 20. 
2. Art. 6 Abs. 2 GG

a) Schutz des Kindes

Die anonyme Kindesabgabe berührt nicht nur das Recht des Kindes auf Kenntnis seiner Abstammung, sondern sie unterbindet auch die Sorge durch seine leiblichen Eltern.

Dieses Recht schützt Art. 6 Abs. 2 S. 1 GG. In Rechtsprechung und Literatur wurde dieser zwar bislang nur als ein Recht der Eltern angesehen, das diese im Interesse des Kindes ausüben müssen, ohne dass dadurch das Kind zum Grundrechtsträger werde. ${ }^{31}$ Das Bundesverfassungsgericht hat die Bestimmung aber nunmehr zu einem eigenständigen Grundrecht des Kindes aufgewertet, das ihm ein Recht auf Umgang mit beiden Elternteilen auch gegen deren Willen vermittle: „Die persönliche Beziehung zu seinen Eltern, ihre Pflege, Hilfe wie Zuwendung tragen wesentlich dazu bei, dass sich das Kind zu einer Persönlichkeit entwickeln kann, die sich um ihrer selbst geachtet weiß und sich selbst wie andere zu achten lernt." Daher stelle die Verweigerung jeglichen Umgangs mit dem Kind und damit die Loslösung von einer persönlichen Bindung zu diesem einen maßgeblichen, für das Kind und seine Entwicklung entscheidenden Entzug elterlicher Verantwortung und zugleich die Vernachlässigung eines wesentlichen Teils der in Art. 6 Abs. 2 Satz 1 GG den Eltern auferlegten Erziehungspflicht dar. ${ }^{32}$

Im konkreten Fall hat das Bundesverfassungsgericht zwar das Recht des Kindes zurücktreten lassen, weil es die Umgangspflicht in der konkreten Konstellation als kontraproduktiv ansieht, betont aber den grundsätzlichen Vorrang des Interesses des Kindes am persönlichen Kontakt mit beiden Eltern vor dem elterlichen Wunsch, auf diesen zu verzichten: „Denn als gewichtige Basis für den Aufbau und Erhalt einer persönlichen familiären Beziehung ebenso wie für das Empfangen elterlicher Unterstützung und Erziehung ist der Umgang eines Kindes mit seinen Eltern für seine Persönlichkeitsentwicklung von maßgeblicher Bedeutung und trägt grundsätzlich zu seinem Wohle bei." ${ }^{33}$ Das ist auch deshalb außerordentlich wichtig, weil es damit überhaupt nicht mehr darauf ankommt, wie sich die Väter von anonym abgegebenen Kindern im Vorfeld der Geburt verhalten haben. Das Kind selbst hat diesen Anspruch auf väterliche Sorge und auf Kontakt mit seinem Vater.

\section{b) Schutz des leiblichen Vaters}

Grundsätzlich wird auch der leibliche Vater durch Art. 6 Abs. 2 GG geschützt. Zwar soll die biologische Vaterschaft für sich gesehen nicht in den Schutzbereich fallen. ${ }^{34}$ Der Vater hat aber ein Recht auf verfahrensrechtlichen Zugang zum Elternrecht, ${ }^{35}$ d.h.

31 BVerfGE 28, 104 (112); 61, 18 (27); G. Robbers, in: H. von Mangoldt/F. Klein/C. Starck (Hrsg.), Kommentar zum Grundgesetz Bd. I, 5. Aufl. 2005, Art. 6 Rn. 182; A. Schmitt-Kammler, in: M. Sachs (Hrsg.), Grundgesetz. Kommentar, 4. Aufl. 2007, Art. 6 Rn. 48.

32 BVerfG, NJW 2008, 1287 (1288f.).

33 BVerfG, NJW 2008, 1287 (1290).

34 BVerfGE 108, 82 (99); kritisch etwa W. Roth, Vaterschaftsanfechtung durch den biologischen Vater, NJW 2003, 3153 (3154).

35 BVerfGE 108, 82 (104ff.). 
er muss auch in die Lage versetzt werden, seine Vaterschaft anzuerkennen oder gerichtlich feststellen zu lassen (§ $1592 \mathrm{Nr} .2$ und 3 BGB). ${ }^{36}$

\section{Beeinträchtigungen der Grundrechte}

Da grundsätzlich nur der Staat Adressat der Grundrechte ist (Art. 1 Abs. 3 GG), muss für die Prüfung des Eingriffs die Rolle des Staates definiert werden.

Die derzeit praktizierte Duldung der Angebote anonymer Kindesabgabe verstößt schon gegen einfaches Recht. ${ }^{37}$ Das ist kein spezifisch grundrechtliches Problem. Vielmehr muss nur das einfache Recht vollzogen werden; ${ }^{38}$ geschieht dies nicht, missachten die zuständigen Behörden ihre verfassungsrechtliche Bindung an Recht und Gesetz (Art. 20 Abs. 3 GG).

Anknüpfungspunkt für eine Beeinträchtigung könnte aber die Zulassung anonymer Kindesabgaben sein. Auch durch eine Zulassung würde der Staat nicht selbst in das allgemeine Persönlichkeitsrecht (Art. 2 Abs. 1 i.V.m. Art. 1 Abs. 1 GG) und das Elternrecht (Art. 6 Abs. 2 GG) eingreifen, sondern dem abgebenden Elternteil die Befugnis einräumen, Kind und Vater durch die anonyme Abgabe von der Wahrnehmung dieser Grundrechte auszuschließen. Doch schützen die Grundrechte nicht nur vor aktiven Eingriffen des Staates. Sie sind also nicht nur Abwehrrechte, sondern erlegen ihm auch die Pflicht auf, den betroffenen Grundrechtsträger vor den Eingriffen Dritter zu schützen. Das Bundesverfassungsgericht hat die Schutzdimension der Grundrechte in den Urteilen zum Schutz des ungeborenen Lebens vor Schwangerschaftsabbrüchen und damit zu Art. 2 Abs. 2 S. 1 GG entwickelt, ${ }^{39}$ leitet aber letztlich aus allen Grundrechten und insbesondere auch aus dem allgemeinen Persönlichkeitsrecht ${ }^{40}$ staatliche Schutzpflichten $\mathrm{ab}$, aus denen wiederum Schutzrechte des Grundrechtsträgers folgen. ${ }^{41}$ Das Unterlassen von Schutz kann damit grundsätzlich ebenso ein Eingriff in ein Grundrecht sein wie eine aktive staatliche Beeinträchtigung. ${ }^{42}$ Die Voraussetzungen für die Auslösung der staatlichen Schutzpflicht sind zwar umstritten; in der Literatur wird sie bisweilen von einer gewissen Mindestintensität der Beeinträchtigung abhängig gemacht. ${ }^{43}$ Doch ist das weniger für die Entstehung, als vielmehr für die Reichweite der Schutzpflicht von Bedeutung und damit für die Frage des gesetzgeberischen Gestaltungsspielraums, ${ }^{44}$ die im Rahmen der verfassungsrechtlichen Rechtfertigung zu thematisieren ist. ${ }^{45}$ Sie ist

36 Zum neuen Verfahren der Abstammungsklärung des Vaters nach §1598a BGB: D. Schwab, Familienrecht, 16. Auflage 2008, Rn. 554-558.

37 Vgl. oben B.

38 Dazu Mielitz (Fn. 3), S. 269ff. und Wolf (Fn. 16), S. 118.

39 BVerfGE 39, 1 (36ff.); 88, 203 (251); vgl. etwa G. Hermes, Das Grundrecht auf Schutz von Leben und Gesundheit, 1987, S. 43ff.

40 Dazu BVerfGE 54, 148 (153); 79, 256 (268); 99, 185 (194f.).

41 B. Pieroth/B. Schlink, Grundrechte. Staatsrecht II, 24. Aufl. 2008, Rn. 82/83, 94ff.

42 H. D. Jarass, in: H. D. Jarass/B. Pieroth, Grundgesetz. Kommentar, 9. Aufl. 2008, Vorb. vor Art. 1, Rn. 31ff.

43 K. Stern, Das Staatsrecht der Bundesrepublik Deutschland Bd. III/1, 1988, S. 740.

44 W. Cremer, Freiheitsgrundrechte, 2003, S. 288; H.-U. Erichsen, Grundrechtliche Schutzpflichten in der Rechtsprechung des Bundesverfassungsgerichts, Jura 1997, 85 (87).

45 Vgl. unten E. I. 
zudem keine Besonderheit des Schutzrechts, sondern gilt auch für das Abwehrrecht. Daher betont auch der EGMR in der Entscheidung Odièvre/Frankreich, dass sich die Grenze zwischen positiven und negativen Verpflichtungen des Staates nur schwer ziehen lasse und die anwendbaren Grundsätze daher in der Abwehr- und Schutzkonstellation ähnlich seien: Jeweils gehe es darum, einen gerechten Ausgleich der widerstreitenden Interessen herbeizuführen. ${ }^{46}$

Der Staat greift also in die Grundrechte des Kindes und des Vaters ein, wenn er es zulässt, dass Mütter ihre Kinder anonym abgeben und diesen damit Informationen über ihre Herkunft und den Vätern über ihre Nachkommenschaft vorenthalten. ${ }^{47}$

\section{Verfassungsrechtliche Rechtfertigung der Grundrechtseingriffe}

Der mit der Legalisierung der anonymen Kindesabgabe einhergehende Eingriff ist verfassungswidrig, wenn damit das von Verfassungs wegen notwendige Mindestmaß an Schutz für die Grundrechte des anonym abgegebenen Kindes und seines Vaters unterschritten würde.

\section{Maßstab für die Erfüllung der Schutzpflicht}

Die Formeln des Bundesverfassungsgerichts zum Maßstab für die Erfüllung der grundrechtlichen Schutzpflicht sind uneinheitlich. In vielen Entscheidungen beschränkt es sich auf die Prüfung, ob ,die öffentliche Gewalt Schutzvorkehrungen entweder überhaupt nicht getroffen hat oder die getroffenen Regelungen und Maßnahmen gänzlich ungeeignet oder völlig unzulänglich sind, das gebotene Schutzziel zu erreichen, oder erheblich dahinter zurückbleiben." 48 Andere Entscheidungen nehmen hingegen eine Inhaltskontrolle des Schutzkonzeptes vor. Zur Bestimmung des grundrechtlich geforderten Minimalschutzes greifen sie auf das Untermaßverbot zurück, das, als Pendant zum abwehrrechtlichen Übermaßverbot, die Funktion des Grundsatzes der Verhältnismäßigkeit in der Schutzkonstellation übernehmen soll. ${ }^{49}$ In der 2. Entscheidung zum Recht des Schwangerschaftsabbruchs heißt es: „Notwendig ist ein - unter Berücksichtigung entgegenstehender Rechtsgüter - angemessener Schutz; entscheidend ist, dass er als solcher wirksam ist. Die Vorkehrungen, die der Gesetzgeber trifft, müssen für einen angemessenen und wirksamen Schutz ausreichend sein und zudem auf sorgfältigen Tatsachenermittlungen und vertretbaren Einschätzungen beruhen. "50

Trotz der Uneinheitlichkeit der Formeln zur Bestimmung der Schutzpflicht sind die Unterschiede hinsichtlich der Kontrolldichte letztlich graduell, nicht kategorisch: In allen Entscheidungen bekennt sich das Bundesverfassungsgericht zur grundsätzlichen Gestaltungsfreiheit des Gesetzgebers. Es legt daher auch in keiner Entscheidung fest,

46 EGMR, NJW 2003, 2145 (2147 Nr. 40).

47 Ebenso Elbel (Fn. 7), S. 258; Mielitz (Fn. 3), S. 175.

48 BVerfGE 92, 26 (46); vgl. ferner BVerfGE 77, 170 (214); 79, 174 (202); 85, 191 (212).

49 M. Möstl, Probleme der verfassungsprozessualen Geltendmachung gesetzgeberischer Schutzpflichten, DÖV 1998, 1029 (1038f.).

50 BVerfGE 88, 203 (254); vgl. bereits BVerfGE 39, 1 (44, 51 f.). 
wie genau der Schutz des Grundrechts ausgestaltet sein muss, sondern umschreibt lediglich das Schutzniveau, das nicht unterschritten werden darf. ${ }^{51}$ Weder Über- noch Untermaß kennen nur ein Maß, sondern grundsätzlich eine Vielzahl verfassungsrechtlich zulässiger Schutzvarianten, unter denen der Gesetzgeber wählen kann. ${ }^{52}$ Dass das Bundesverfassungsgericht das Schutzkonzept des Gesetzgebers mal mehr, mal weniger intensiv prüft und damit auch den notwendigen Mindestschutz in unterschiedlicher Detailliertheit bestimmt, liegt daran, dass Art, Nähe und Ausmaß der drohenden Gefahr, die schon vorhandenen Regelungen und getroffenen Maßnahmen, sowie schließlich die mit dem grundsätzlichen Schutzauftrag kollidierenden Interessen jeweils unterschiedlich sind. ${ }^{53}$

\section{Die Bestimmung des verfassungsrechtlich zwingenden Schutzniveaus}

$\mathrm{Zu}$ prüfen ist daher, ob die Legalisierung der anonymen Kindesabgabe das durch die Art. 2 Abs. 1 i.V.m. Art. 1 Abs. 1 und Art. 6 Abs. 2 GG vorgegebene Mindestmaß an staatlichem Schutz unterschreiten würde. Dabei ist zu berücksichtigen, dass die Ermöglichung anonymer Kindesabgaben als Beitrag zur Sicherung der Persönlichkeitsrechte der Mutter, sowie zur Verhinderung von Schwangerschaftsabbrüchen und Kindstötungen verstanden wird. Die Frage nach dem verfassungsrechtlich notwendigen Mindestschutz lässt sich daher nicht abstrakt, sondern nur konkret unter Berücksichtigung der Ziele beantworten, die mit einer Legalisierung der anonymen Kindesabgabe verfolgt werden. Gegenstand der Prüfung ist daher nicht die begehrte Erfüllung der Schutzpflicht, sondern die Maßnahme, die das Schutzniveau absenkt, ${ }^{54}$ also die Legalisierung der anonymen Kindesabgabe. Damit kann, ebenso wie bei dem auf Eingriffsminimierung zielenden Abwehrrecht, auch bei der Schutzpflicht der Grundsatz der Verhältnismäßigkeit aktiviert werden, wobei es hier nicht um die Frage geht, ob der Staat zu viel in die Grundrechte eingegriffen hat, sondern darum, ob er sie zu wenig geschützt hat. ${ }^{55}$

\section{a) Geeignetheit}

Ein Ziel der Legalisierung der anonymen Kindesabgabe könnte das durch das allgemeine Persönlichkeitsrecht (Art. 2 Abs. 1 i.V.m. Art. 1 Abs. 1 GG) geschützte Interesse der Mutter an Anonymität sein. Dieses Interesse allein kann aber die lebenslangen Rechtsverluste insbesondere des Kindes nicht legitimieren, weil die Eltern mit der Zeugung die Elternverantwortung gegenüber dem Kind übernommen haben. ${ }^{56}$ Die Garantie

51 Vgl. die Nachweise bei F. Hufen, Staatsrecht II. Grundrechte, 2007, § 13 Rn. 18.

$52 \mathrm{Zu}$ diesem Korridor zwischen dem zulässigen Höchstmaß des Eingriffs und dem notwendigen Mindestmaß des Schutzes C. Calliess, Rechtsstaat und Umweltstaat, 2001, S. 457 f. und $T$. Aubel, Der verfassungsrechtliche Mutterschutz, 2003, S. $262 \mathrm{f}$.

53 W. Durner, Verfassungs- und Völkerrechtsfragen der anonymen Geburt, ZG 2005, 243 (250f.).

54 L. Michael/M. Morlok, Grundrechte, 2008, Rn. 628.

55 Vgl. dazu die unterschiedlichen Ansätze bei Calliess (Fn. 52), S. 459 f.; Hermes (Fn. 39), S. 253ff. und Michael/Morlok (Fn. 54), Rn. 627ff.

56 BVerfG, NJW 1988, 3010 (3010). 
der Anonymität ist daher nur ein Mittel für einen weiteren grundsätzlich verfassungslegitimen Zweck: der Schutz von Leben und körperlicher Unversehrtheit des Kindes (Art. 2 Abs. 2 S. 1 GG). Dabei geht es vor allem um den Schutz des neugeborenen Lebens. Der Beitrag für den Schutz des ungeborenen Lebens dürfte hingegen nicht sonderlich hoch sein. Nach $\S 218$ a Abs. 1 StGB sind nämlich ärztlich durchgeführte Schwangerschaftsabbrüche innerhalb von zwölf Wochen nach der Empfängnis straflos, wenn die Schwangere eine Beratungsbescheinigung ( $\$ 219$ Abs. 2 S. 2 StGB) vorlegt. Wenn eine Mutter wegen ihrer besonderen psychosozialen Lebenssituation das Kind nicht großziehen möchte, aber gleichwohl bereit ist, es auszutragen, kann sie das Kind auf dem Weg der regulären Adoption abgeben.

Es kommt also entscheidend darauf an, ob Angebote der anonymen Kindesabgabe einen Beitrag dazu leisten, die schutzlose Aussetzung und Tötung Neugeborener zu verhindern. ${ }^{57}$ Befürworter und Gegner sind sich immerhin darin einig, dass die Datenlage dürftig ist. ${ }^{58}$ Beide Seiten arbeiten weitgehend mit eigenen Erhebungen, wobei Medienberichte die wichtigste Informationsquelle bilden. Wie nicht anders zu erwarten, weichen die Zahlen ebenso voneinander ab wie die daraus gezogenen Schlussfolgerungen. ${ }^{59}$ Es dominieren Erfahrungsberichte, die oftmals mit Vermutungen und Spekulationen angereichert werden.

Nach Auffassung ihrer Befürworter verhindern Angebote der anonymen Kindesabgabe die Aussetzung und Tötung Neugeborener. ${ }^{60}$ Sie sollen diejenigen Frauen ansprechen, die aufgrund ihrer besonderen persönlichen Situation, ihres familiären Umfelds und ihres psychosozialen Zustands für die herkömmlichen Hilfskonzepte nicht erreichbar sind. Das sind vor allem Minderjährige, Frauen in Ausbildung, Drogenabhängige und ausländische oder illegal in Deutschland lebende Frauen. ${ }^{61}$ Häufig soll es sich dabei um Frauen handeln, die sich gerade wegen ihrer schwierigen persönlichen Situation der Tatsache der Schwangerschaft, bewusst oder unbewusst, verschließen und daher auf die Geburt nicht vorbereitet sind. Adoptionen seien für diesen Personenkreis keine Alternative, weil sie stets mit der von außen an sie herangetragenen Stigmatisierung einer Mutter, die ihr Kind abgegeben hat, leben müssten. Um das zu verhindern, bleibe nur die Anonymität.

Die Gegenthese lautet, dass die Angebote anonymer Kindesabgabe gerade diejenigen Frauen, bei denen die Gefahr der Aussetzung/Tötung des Kindes besteht, nicht er-

57 Vgl. dazu etwa die Untersuchungen des Bayerischen Staatsministeriums für Arbeit und Sozialordnung, Familie und Frauen: „Anonyme Geburt“ - „Moses-Projekt“ in Bayern. Eine Machbarkeitsstudie, 2007, sowie von M.-B. Singer, Babyklappen und anonyme Geburt, 2008.

58 Vgl. zum Problem der empirischen Unsicherheit auch Durner (Fn. 53), S. 255ff.

59 Bayerisches Staatsministerium (Fn. 57), S. 25 f.

60 Vgl. zum Folgenden die Begründungen der Gesetzentwürfe oben Fn. 19 und 20 sowie etwa R. Hepting, „Babyklappe“ und ,anonyme Geburt“, FamRZ 2001, 1573 (1577) sowie J. Heyers, Zivilrechtliche Institutionalisierung anonymer Geburten, JR 2003, 45 (46) unter Berufung auf französische Erfahrungen.

61 Bayerisches Staatsministerium (Fn. 57), S. 23. 
reicht. ${ }^{62}$ Kindstötungen würden von Frauen begangen, die unter erheblichen Persönlichkeitsstörungen leiden. Kriminologische Untersuchungen zeigten, dass Neugeborenentötungen meist im Affekt begangen würden. Es handele sich um Mütter, die die Schwangerschaft verdrängen, von der Geburt daher überrascht werden und das Kind dann in der ersten Panik töten. Diese Frauen würden weder die regulären Angebote der Schwangerenkonfliktberatung noch die Angebote anonymer Kindesabgabe wahrnehmen. Beides setze nämlich ein Minimum an Beschäftigung mit der bevorstehenden Geburt voraus. Die Angebote anonymer Kindesabgabe schließen daher, so die Vermutung, keine Lücke, sondern wecken bei einem Personenkreis, der grundsätzlich in der Lage ist, Konflikte zu lösen, und daher für die regulären Beratungsangebote erreichbar ist, eine zuvor nicht vorhandene Nachfrage.

Als gemeinsame Schnittmenge in diesem Spektrum von Meinungen kann man nur die recht allgemeine Erkenntnis festhalten, dass nicht alle Kinder, die anonym abgegeben werden, beim Fehlen dieses Angebotes getötet worden wären, dass aber umgekehrt auch nicht auszuschließen ist, dass das Angebot einer anonymen Geburt Leben gerettet hat. Auch die Befürworter von Angeboten anonymer Kindesabgaben haben allerdings keinen einzigen solchen Fall dokumentiert, wie es überhaupt nach wie vor an einer umfassenden bundesweiten Erhebungen fehlt. ${ }^{63}$ Zwar hat der Gesetzgeber bei der Frage der Geeignetheit eines Schutzkonzeptes einen weiten Einschätzungsspielraum ${ }^{64}$ und er ist, wenn er die zugänglichen Erkenntnisquellen ausgeschöpft hat, befugt, Entscheidungen auch unter den Bedingungen der Ungewissheit zu fällen. ${ }^{65}$ Jedenfalls die Bundesregierung geht aber davon aus, dass noch weitere Daten erhoben werden müssen und können. ${ }^{66}$

Es erscheint zwar fraglich, wie diese Daten erhoben werden sollen, weil nur diejenigen Frauen befragt werden können, die später auf die Anonymität verzichtet haben und damit eine Personengruppe mit einem vermutlich zu starken Bias. Auch über die Entwicklung der Tötungen Neugeborener gibt es keine belastbaren Zahlen, da diese seit der Abschaffung von $\S 217$ StGB in den Kriminalstatistiken nicht mehr separat ausgewiesen werden. ${ }^{67}$ Abgesehen davon werden glücklicherweise so wenige Neugeborene getötet, dass man aus veränderten Zahlen kaum verlässliche Kausalzusammenhänge ableiten könnte; feststellen lässt sich allerdings, dass auch dort, wo Angebote anonymer Kin-

62 U. Herpich-Behrens, Was brauchen Mütter in höchster Not wirklich?, Die Sicht des Landesjugendamtes Berlin, in: terre des hommes Deutschland e. V. (Hrsg.), Babyklappe und anonyme Geburt - ohne Alternative?, 2003, S. 147ff.; und A. Rohde, Welche Mütter töten ihre Kinder?, ebda, S. 131ff. sowie die Stellungnahmen von terre des hommes (http://www.terredeshommes.de/content/themen/weitere/babyklappe/position_tdh.htm).

63 Als Desiderat vermerkt auch in der Untersuchung des Bayerischen Staatsministeriums (Fn. 57), S. 55 f.

64 Vgl. oben I.

65 BVerfGE 50, 290 (332).

66 BT-Drucks. 16/7220, S. 9.

67 C. Gremmel, Anonyme Geburt \& anonyme Abgabe von Kindern. Hilfe oder Risiko? Ein deutsch-französischer Vergleich, in: G. Zenz (Hrsg.), Traumatische Kindheiten. Beiträge zum Kinderschutz und zur Kinderschutzpolitik aus erziehungswissenschaftlicher und rechtswissenschaftlicher Perspektive, 2004, S. 107 (111); vgl. ferner die Studie von M. Bauermeister, Die Tötung Neugeborener unter der Geburt (Kindstötung § 217 StGB). Eine bundesweite Verbundstudie für die Jahre 1980-1989, 1994. 
desabgabe zur Verfügung stehen, nach wie vor Neugeborene ausgesetzt und getötet werden. ${ }^{68}$

Dennoch könnte der Gesetzgeber, der anonyme Geburten unter bestimmten Voraussetzungen zulässt, mit einer solchen Untersuchung immerhin den Nachweis führen, dass er alles zur Ermittlung der tatsächlichen Gegebenheiten Mögliche und Zumutbare getan hat. Kommt diese Untersuchung zu dem belastbaren Ergebnis, dass es Fälle gibt, in denen die Angebote anonymer Kindesabgabe das Leben eines Kindes retten können, wäre die Eignung grundsätzlich zu bejahen. Bedenken begegnet hingegen der Vorschlag, die Wissenslücken mit einem Experimentiergesetz zu schließen. ${ }^{69}$ Denn die empirische Unsicherheit beruht nicht auf dem Fehlen einer gesetzlichen Grundlage (im Gegenteil wird die anonyme Kindesabgabe ja auch ohne gesetzliche Grundlage praktiziert), sondern auf der Anonymität, die das Gesetz nicht beseitigen, sondern perpetuieren würde. ${ }^{70}$

\section{b) Erforderlichkeit}

Im Rahmen der Erforderlichkeit rückt das Verhältnis zwischen den beiden Formen der anonymen Kindesabgabe in den Mittelpunkt. Zu prüfen ist nämlich, ob es ein gleichermaßen geeignetes Mittel gibt, das die betroffenen Grundrechte weniger stark einschränkt. ${ }^{71}$

Tatsächlich besteht in mehrfacher Hinsicht ein qualitativer Unterschied zwischen den beiden Angeboten. Anonyme Geburten werden medizinisch betreut, Babyklappen können hingegen erst nach einer Entbindung genutzt werden. Bei Babyklappen lässt sich zudem, anders als bei der anonymen Geburt, nicht feststellen, wer das Kind abgibt. Nicht immer ist es die Mutter, die das Kind einlegt, und es lässt sich noch nicht einmal ausschließen, dass das Kind gegen ihren Willen abgegeben wird. Hinzu kommt, dass keine Kontaktaufnahme zur abgebenden Person und damit keine auf Aufgabe der Anonymität gerichtete Beratung stattfinden kann. Auch ist die Hemmschwelle, sich des Kindes zu entledigen, wesentlich geringer, wenn die Betroffene noch nicht einmal gezwungen ist, Beweggründe für ihre Entscheidung darzulegen. Die Möglichkeiten, einen Ausgleich mit den Interessen von Kind und nicht abgebendem Elternteil herbeizuführen, sind daher bei der anonymen Geburt ungleich größer als bei der Babyklappe, weil eine medizinische und sozialpsychologische Kontaktaufnahme mit der Mutter möglich ist. Aus diesem Grund wird in Frankreich auch nur die anonyme Geburt (,accouchement sous X“), nicht aber die Abgabe in Babyklappen praktiziert. ${ }^{72}$

Allenfalls könnte man fragen, ob anonyme Geburten weniger geeignet sind, ihr Ziel zu erreichen, weil werdende Mütter anonyme Geburten wegen der Notwendigkeit der persönlichen Kontaktaufnahme nicht in Anspruch nehmen. Dass aber eine Mutter ein

68 S. Neuheuser, Babyklappenkonzepte - guter Wille wider Rechtsordnung, ZfL 2002, 10 (13) sowie die Entscheidung BGH 4 StR 105/08 v. 19.6.2008 (Tötung eines Neugeborenen trotz Kenntnis der Mutter von einer Babyklappe).

69 Durner (Fn. 53), S. 258.

70 Kritisch auch D. Elbel, Anonyme Geburten und Babyklappen, KritV 2007, 293 (298).

71 BVerfGE 67, 157 (177); 68, 193 (219); 92, 262 (273).

72 Vgl. dazu Mielitz (Fn. 3), S. 33ff. 
Kind nur deshalb tötet, weil zwar ein Angebot anonymer Abgabe, nicht aber eine Babyklappe zur Verfügung steht, ist hochspekulativ und daher irrelevant. Eine Legalisierung von Babyklappen wäre daher schon wegen fehlender Erforderlichkeit und unabhängig von der Frage der Geeignetheit im Allgemeinen verfassungswidrig. ${ }^{73}$

\section{c) Angemessenheit}

Der mit der Legalisierung der anonymen Geburt einhergehende Schutzverlust muss schließlich in einem angemessenen Verhältnis zu den positiven Effekten für den Schutz des neugeborenen Lebens stehen.

\section{aa) Unabwägbarkeit des Lebensrechts (Art. 2 Abs. 2 GG)?}

Zunächst kann man fragen, ob die beiden Schutzkonzepte überhaupt der gegenseitigen Abwägung zugänglich sind. Es wird nämlich gesagt, dass die Wahrnehmung der Persönlichkeitsrechte und der Umgangsrechte stets das Überleben des Kindes voraussetze. ${ }^{74}$ Diese intrapersonale Abwägung lässt sich leicht widerlegen, weil, soweit ersichtlich, jedenfalls niemand behauptet, dass alle anonym geborenen Kinder ohne das Angebot anonymer Geburt nicht mehr leben würden.

Es geht also gerade um die Frage, ob es legitim ist, den Grundrechtsschutz von Kindern und Vätern einzuschränken, um das Leben anderer Kinder zu retten, d. h. um einen interpersonalen Konflikt zwischen unterschiedlichen Grundrechtsträgern. Aber auch insoweit wird der Sinn, ja überhaupt die Legitimität einer Abwägung bestritten: Allein die Chance, nur ein einziges Kind zu retten, sei doch ausreichend, um das Angebot anonymer Kindesabgaben zu legitimieren. ${ }^{75}$ Auch diese These stellt die generelle Abwägbarkeit der beiden Schutzkonzepte in Frage, indem sie unausgesprochen einen Vorrang des Grundrechts auf Leben vor anderen Grundrechten postuliert. Eine solche abstrakte Rangordnung der Grundrechte ist dem Grundgesetz außerhalb der als unverfügbar vorgegebenen Menschenwürde (Art. 1 Abs. 1 GG) und dem damit eng zusammenhängenden absoluten Verbot der Todesstrafe (Art. $102 \mathrm{GG}$ ) allerdings fremd. ${ }^{76}$ Richtig ist zwar, dass generell eine besonders strenge Prüfung geboten ist, wenn Leben und Gesundheit zur Abwägung stehen. ${ }^{77}$ Der Gesetzesvorbehalt (Art. 2 Abs. 2 S. 3 GG) belegt aber, dass auch das Grundrecht auf Leben im Einzelfall hinter anderen Schutzgütern zurücktreten muss, beim Schwangerschaftsabbruch etwa hinter dem Persönlichkeitsrecht der Mutter. ${ }^{78}$ Das legitimiert auch den Verzicht auf bei isolierter Betrachtung durchaus mögliche Verbesserungen des Lebensschutzes. So könnte der Gesetzgeber, zur Behebung des Organmangels, jeden Menschen zwingen, post mortem seine Organe zu spenden, soweit das medizinisch möglich und sinnvoll ist. Er würde damit vermutlich

73 Auch Elbel (Fn. 7), S. 361, hält ein Verbot von Babyklappen für unerlässlich.

74 I. Mittenzwei, Babyklappe und anonyme Geburt, ZRP 2002, 452 (452).

75 Hepting (Fn. 60), S. 1578; T. Müller-Magdeburg, Recht auf Leben - Die anonyme Geburt, FPR 2003, 109 (111).

76 Vgl. etwa R. Alexy, Theorie der Grundrechte, 2. Aufl. 1994, S. 142.

77 Vgl. H. D. Jarass, in: Jarass/Pieroth (Fn. 42), Art. 2 Rn. 96.

78 BVerfGE 88, 203 (254); vgl. E. Benda, Die ,,anonyme Geburt“, JZ 2003, 534 (537). 
verhindern, dass Menschen auf den Wartelisten nur deshalb sterben, weil für sie kein Spenderorgan zur Verfügung steht. Aber er verzichtet darauf mit Rücksicht auf die Verfügungsrechte des Einzelnen über seinen Körper.

Es gibt daher im Hinblick auf das Grundrecht auf Leben kein generelles Abwägungsverbot.

\section{bb) Kriterien für die Abwägung}

(1) Struktur des Grundrechtskonflikts

In der Grundrechtsdogmatik spricht man von Kollisionen, wenn ,,vermittlungsbedürftige, weil potentiell oder aktuell konfligierende Freiheitsausübungsansprüche verschiedener Grundrechtsträger"79 bestehen. Kollisionen sind also Konflikte zwischen zwei gleichermaßen grundgesetzlich gewährleisteten Freiheitsbetätigungen, die aufeinander prallen und daher nach Maßgabe der Gesetzesvorbehalte der Grundrechte und des Grundsatzes der Verhältnismäßigkeit zu einem Ausgleich gebracht werden müssen: der Informationsanspruch des die Anbringung einer Parabolantenne begehrenden türkischen Mieters (Art. 5 Abs. 1 S. 1 Hs. 2 GG) mit dem Eigentumsgrundrecht des Vermieters (Art. 14 Abs. 1 GG), ${ }^{80}$ die Meinungs- und Pressefreiheit (Art. 5 Abs. 1 S. 1 Hs. 1 und S. 2 Var. 1 GG) mit den Persönlichkeitsrechten der von Meinungsäußerungen und Presseveröffentlichungen negativ Betroffenen ${ }^{81}$ und das Persönlichkeitsrecht der Mutter, den Namen des Vaters zu verschweigen mit dem Anspruch des Kindes, diesen zu kennen. ${ }^{82}$ Charakteristikum der Kollision ist also ein grundrechtlich geschütztes Handeln, das in einer konkreten Situation Konfliktpotential auslöst; die Beteiligten weisen dabei jeweils eine qualifizierte Nähe zu dem Konflikt auf, die sie von anderen Grundrechtsträgern abhebt. Die gegenläufigen Interessen sind durch das Zusammentreffen auf eine Art und Weise miteinander verflochten, dass ein Ausgleich erforderlich ist. Diese Verflechtung legitimiert staatliche Regulierung und damit auch eine Relativierung des grundrechtlichen Schutzanspruches im Einzelfall.

Das Kind, das anonym abgegeben wurde, weist nun aber mit seinem Anspruch auf Freilegung seiner Wurzeln keine qualifizierte Nähe zum Problem der Tötung des ungeborenen/neugeborenen Lebens auf. Entsprechendes gilt für den Vater des Kindes, der von seinem Kind nicht erfährt oder jedenfalls keinen Umgang mit diesem haben darf, damit andere Mütter oder Väter ihre Kinder nicht töten. Der Konflikt wird also erst durch die Knüpfung von Kausalketten konstruiert, deren Haltbarkeit jedenfalls unsicher ist. ${ }^{83}$ Die Grundrechte sind hier mithin nicht dergestalt miteinander verflochten, dass es eines Ausgleichs bedarf. Damit ist die Legitimation für den Grundrechtseingriff zweifelhaft: Während sie sich bei einer Grundrechtskollision aus der Beteiligung der Grundrechtsträger an dem konkreten Konflikt ergibt, ist sie problematisch, wenn der Grundrechtseingriff unbeteiligte Personen trifft, die keinerlei qualifizierten Bezug zu dem

79 H. Dreier, in: H. Dreier (Hrsg.), Grundgesetz. Kommentar Bd. I, 2. Aufl. 2004, Vorb., Rn. 157; ferner insbesondere Pieroth/Schlink (Fn. 41), Rn. 314ff.

80 BVerfGE 90, 27.

81 BVerfGE 93, 266.

82 BVerfGE 96, 56.

83 Vgl. dazu nochmals oben 1. 
konkreten Konflikt aufweisen. Die Legalisierung anonymer Geburten würde einen grundrechtlichen Kollateralschaden produzieren: Um das Leben einzelner Kinder zu schützen, wird anderen Kindern ihre Abstammung vorenthalten und werden die Beziehungen zwischen Kindern und Eltern abgeschnitten. Das Bundesverfassungsgericht sieht diese zufällige Mithaftung in seiner Entscheidung zur Verfassungsmäßigkeit der Rasterfahndung kritisch: „Grundrechtseingriffe, die sowohl durch Verdachtslosigkeit als auch durch eine große Streubreite gekennzeichnet sind - bei denen also zahlreiche Personen in den Wirkungsbereich einer Maßnahme einbezogen werden, die in keiner Beziehung zu einem konkreten Fehlverhalten stehen und den Eingriff durch ihr Verhalten nicht veranlasst haben - weisen grundsätzlich eine hohe Eingriffsintensität auf [...]. Denn der Einzelne ist in seiner grundrechtlichen Freiheit umso intensiver betroffen, je weniger er selbst für einen staatlichen Eingriff Anlass gegeben hat." 84

\section{(2) Einfluss der Legalisierung der anonymen Geburt auf die Schutzkonzepte}

Die Streubreite allein macht den Eingriff zwar noch nicht verfassungswidrig. Sie enthält aber eine Aussage über die Intensität des Eingriffs, der ein angemessen hoher Zuwachs an Rechtsgüterschutz gegenüberstehen muss. Dazu ist zu untersuchen, welchen Einfluss eine Legalisierung der anonymen Geburt auf die beiden Schutzkonzepte hat. Zu prüfen ist m. a. W., wie sich die Zulassung der anonymen Geburt auf den Schutz des neugeborenen Lebens auf der einen Seite und die Persönlichkeits- und Elternrechte auf der anderen Seite auswirken würde.

Hier bestehen verfassungsrechtlich relevante Diskrepanzen. Es dürfte Einigkeit darüber bestehen, dass der Gesetzgeber von Verfassungs wegen nicht dazu verpflichtet ist, anonyme Geburten zuzulassen, um seiner Schutzpflicht aus Art. 2 Abs. 2 S. 1 GG nachzukommen. Anonyme Geburten mögen, ihre Wirksamkeit unterstellt, ein kleiner Baustein im Schutzkonzept für das neu- und ungeborene Leben sein, werden aber auch von ihren Befürwortern nur als Ausnahmefall angesehen. Hingegen bedeutet die Zulassung der anonymen Geburt einen erheblichen Eingriff in die familienrechtlichen Eltern-KindBeziehungen. Diese Beziehungen entstehen zwar teils, wie bei der Abstammung ( $\$ 1591$ BGB), qua Gesetz und können daher durch privatautonome Entscheidungen nicht beseitigt werden. Sie laufen aber leer, denn das Kind, das seine Mutter und seinen Vater nicht kennt, kann die aus der Abstammung fließenden Rechte (Unterhalt, Pflege und Erziehung, Erbrecht) nicht mehr wahrnehmen. Faktisch wird es gegenüber seinen leiblichen Eltern familienrechtlich rechtlos gestellt. Der Staat würde anonym bleibende Personen in die Lage versetzen, über fundamentale Familienrechte der Kinder zu disponieren, ohne Verfahren, ohne Begründungszwang und besondere Voraussetzungen. Das reguläre Adoptionsverfahren mit seinen Beteiligungsrechten für den Vater und der Datensicherung durch das PStG würde entwertet.

Besonders bemerkenswert sind auch die Diskrepanzen, was unser Wissen angeht. Während nach wie vor die grundlegenden Daten darüber fehlen, ob anonyme Geburten Leben retten, kann die moderne Adoptionsforschung auf wissenschaftlich fundierte Erkenntnisse über die Gemütslage von Kindern zurückgreifen, die von ihren leiblichen

84 BVerfGE 115, 320 (354). 
Eltern abgegeben wurden. ${ }^{85}$ Danach löst gerade die Trennung zwischen biologischer Herkunft und sozialer Zugehörigkeit das Interesse an der eigenen Abstammung aus. Die Betroffenen nehmen sie als Verlust bewusst wahr, wenn sie ihre Identität bestimmen, das definieren, was sie unverwechselbar macht, auch in Beziehungen zu anderen Menschen. Selbst eine gefestigte soziale Zugehörigkeit zu einer Adoptivfamilie (die ja nicht selbstverständlich ist) kann meist nicht über den Schmerz hinweghelfen, fortgegeben worden zu sein und noch nicht einmal den Grund dafür zu kennen; bisweilen steigert sich das bis hin zu der Vorstellung: „Meine Eltern wollten gerade mich nicht!“; oder: „Wenn ich von so schlechten Eltern abstamme, kann ich auch nur ein schlechtes Kind sein“. Dieser oftmals mit Wut gepaarte Schmerz äußert sich in Zweifeln am Selbstwert, in Beziehungsunfähigkeit und Trennungsängsten. In Interviews bezeichnen betroffene Kinder ihren Geburtstag als den traurigsten Tag ihres Lebens und die Feier mit ihren Adoptiveltern als „Betrug“, weil diese doch gar keinen Beitrag zur Geburt geleistet haben. Wenn Betroffene nach ihrer biologischen Herkunft forschen, so ist damit, bewusst oder unbewusst, die Sehnsucht verbunden, den fundamentalen lebensgeschichtlichen Bruch ungeschehen zu lassen, Hintergründe und Erklärungen für individuelle Verhaltensmuster, aber auch für Erkrankungen und Wege für den möglichen Umgang mit ihnen zu eruieren.

Die Situation des anonym abgegebenen Kindes ist dabei noch schlechter als bei anderen adoptierten Kindern. Diese haben nach Vollendung des 16. Lebensjahres aus $\S 62$ Abs. 1 PStG einen Anspruch auf Einsicht in den Geburtseintrag oder auf Erteilung einer Personenstandsurkunde aus dem Geburtenbuch und nach $\S 9$ b Abs. 2 AdVermG auf Einsichtnahme in die Adoptionsakten, soweit diese seine Herkunft und Lebensgeschichte betreffen. Das regulär adoptierte Kind kann daher, wenn auch erst sehr spät, den Bruch zu Beginn seines Lebens zumindest ansatzweise rekapitulieren und bekommt zumindest die Möglichkeit, den Namen der Mutter (und evtl. des Vaters) zu erfahren und mit dieser sogar Kontakt aufzunehmen. Bei einem anonym geborenen Kind laufen diese Ansprüche hingegen weitgehend leer, weil Mutter und Anbieter ihren personenstandsrechtlichen Verpflichtungen mit Rücksicht auf die Wahrung der Anonymität der Mutter nicht nachgekommen sind. ${ }^{86}$

Man kann damit festhalten: Der Einfluss der anonymen Geburt auf den Schutz des ungeborenen Lebens ist unsicher und, unterstellt es gibt ihn, für das Gesamtkonzept bestenfalls gering, wegen der negativen Auswirkungen auf die vorhandenen Hilfsangebote möglicherweise sogar kontraproduktiv. Die Auswirkungen auf die Persönlichkeitsrechte und Elternrechte insbesondere der Kinder sind hingegen gut dokumentiert und erheblich.

\section{cc) Vertrauliche Geburt?}

Um trotz des eindeutigen Überwiegens der Kinder- und Väterrechte einen verhältnismäßigen Ausgleich herzustellen, wird darüber diskutiert, ob sich Verfahren der anony-

85 Zum Folgenden etwa B. Brand, Adoption und Identität, 2007; C. Hoffmann-Riem, Das adoptierte Kind. Familienleben mit doppelter Elternschaft, 1984; C. Swientek, Wer sagt mir, wessen Kind ich bin? Von der Adoption Betroffene auf der Suche, 1993; I. Wiemann, Pflegeund Adoptivkinder, 7. Aufl. 2003.

86 Vgl. oben B. I. 
men Geburt entwickeln lassen, die den Schutzzielen einer Zulassung anonymer Geburten und den Grundrechten von Kindern und Vätern gleichermaßen gerecht werden.

Vorgeschlagen wird eine sog. vertrauliche Geburt, die eine spätere Durchbrechung der Anonymität der Mutter ermöglichen soll. ${ }^{87}$ Die entscheidende Frage ist aber, ob die Mutter gegen die Offenlegung ihrer Personaldaten ihr Veto einlegen kann, wie das etwa in Frankreich nach wie vor der Fall ist und beim letzten, 2004 in den Bundesrat eingebrachten Gesetzentwurf ${ }^{88}$ vorgesehen war. Bei einem Veto kann es nämlich, wie die sieben in der Entscheidung Odièvre unterlegenen Richter zutreffend feststellen, ,,weder tatsächlich noch rechtlich zu einer Interessenabwägung kommen. [...] Es handelt sich [...] keineswegs um ein gemischtes System, das irgendeinen Ausgleich zwischen den betroffenen Rechten sichert. Das der Mutter zugestandene eindeutige und einfache, Vetorecht' hat zur Folge, dass die im Gesamtsystem der Konvention anerkannten Rechte des Kindes [...] völlig vernachlässigt, ja vergessen werden. Außerdem kann die Mutter in gleicher Weise die Rechte Dritter leer laufen lassen, insbesondere die des leiblichen Vaters oder der Geschwister, die ebenfalls ihre in Art. 8 EMRK garantierten Rechte verlieren." 89

Ein verfassungsgemäßer Ausgleich setzt damit voraus, dass das Kind, ebenso wie andere Adoptivkinder (vgl. §62 Abs. 1 PStG), zu einem späteren Zeitpunkt einen unbedingten, vom Willen der Mutter unabhängigen Anspruch auf Offenlegung seiner Personendaten hat. ${ }^{90}$ Die Mutter würde damit zwar nicht gegenüber ihrem Kind, wohl aber gegenüber ihrem sozialen Umfeld anonym bleiben können - ein Wunsch, den offenbar viele Betroffene äußern. ${ }^{91}$ Fraglich ist aber, welchen Zusatznutzen das Angebot einer vertraulichen Geburt hat. Schon jetzt haben Frauen nach $§ 6$ Abs. 2 SchKG einen Anspruch auf eine anonyme Schwangerschaftskonfliktberatung, und es ist auch nicht grundsätzlich verboten, ein Kind zur Welt zu bringen, ohne das soziale Umfeld daran teilhaben zu lassen. Das Angebot einer vertraulichen Geburt würde die Mutter allerdings von der personenstandsrechtlichen Verpflichtung befreien, den Namen des Vaters zu nennen. ${ }^{92}$ Würde nämlich der Vater spätestens im Zuge des Adoptionsverfahrens einbezogen, könnte Vertraulichkeit gegenüber dem sozialen Umfeld der Mutter nicht mehr garantiert werden. Unter bestimmten Voraussetzungen gesteht ihr die Rechtsprechung das Recht zu schweigen allerdings ohnehin schon jetzt zu, freilich nur im Einzelfall, unter Abwägung der gegenläufigen Interessen und mit der Möglichkeit gerichtlicher, durch Vater oder Kind veranlasster Kontrolle. ${ }^{93}$ Die Zulassung der vertraulichen Geburt wäre demgegenüber ein qualitativer Sprung, weil sie es der Mutter generell und nicht

87 Vgl. dazu insbesondere Elbel (Fn. 7), S. 379ff.; Hepting (Fn. 60), S. 1581 f.

88 BR-Drucks. 682/04, S. 13.

89 EGMR, Gemeinsame abweichende Meinung der Richter Wildhaber, Bratza, Bonello, Loucaides, Cabral Barreto, Tulkens und Pellonpää, NJW 2003, 2145 (2149 Nr. 7); im Hinblick auf die Verkürzung des Beurteilungsspielraums der Mitgliedstaaten kritisch M. Wittinger, Anonyme Geburt - endlich Klarheit?, NJW 2003, 2138 (2139).

90 Vgl. insbesondere Elbel (Fn. 7), S. 384ff.; ferner etwa Bund Deutscher Hebammen (http:// www.dbsh.de/redsys/soztop/userpages/babyklappe.html).

91 Elbel (Fn. 70), S. 301.

$92 \mathrm{Vgl}$. zu dieser Verpflichtung bereits oben B. I.

93 BVerfGE 96, 56 (62); tendenziell für eine weitere Stärkung der Rechte des Kindes $K$. $H$. Muscheler/A. Bloch, Das Recht auf Kenntnis der genetischen Abstammung und der Anspruch des Kindes gegen die Mutter auf Nennung des leiblichen Vaters, FPR 2002, 339 (346ff.). 
nur bei Vorliegen besonderer Umstände gestatten würde, den Vater nicht zu nennen, ohne dass sie sich dafür rechtfertigen muss und ohne dass ihre Entscheidung einer gerichtlichen Kontrolle zugänglich wäre. ${ }^{94}$ Aus der Ausnahme würde die Regel: Die Mutter, die den Vater des Kindes nicht nennen möchte, kann dies rechtmäßigerweise unterlassen. Sie hätte es, nicht anders als bei der absolut anonymen Geburt, in der Hand, den Vater von seinen Rechten (etwa im Rahmen einer Adoption) auszuschließen und zu verhindern, dass das Kind Kenntnis von seiner väterlichen Abstammung erhält. Die personenstandsrechtliche Anzeigepflicht ließe sich damit im Hinblick auf den Vater insgesamt nicht mehr halten. Der Gesetzgeber würde sich der zentralen verfahrensrechtlichen Unterfütterung der Persönlichkeits- und Elternrechte begeben. Das ist keine angemessene Alternative.

\section{Schluss}

Babyklappen und Angebote anonymer Geburt haben auf den ersten Blick einen anziehenden Charme. Wer wollte ernsthaft gegen Angebote sein, die Neugeborenen das Leben retten? Das mag der Grund dafür sein, dass die Behörden derzeit bundesweit vor einem Einschreiten zurückschrecken und den rechtswidrigen Zustand dulden. Man möchte nicht für das nächste getötete Neugeborene verantwortlich gemacht werden.

Bei näherem Hinsehen scheint indes zwischen Glaube und Vernunft eine gewisse Schieflage zu bestehen. Dass Anonymität Leben retten soll, wird behauptet, aber nicht in einem Maße belegt, das es rechtfertigt, Kinder von ihren Wurzeln zu trennen. Gut dokumentiert sind demgegenüber die gravierenden lebenslangen Belastungen anonym abgegebener Kinder, für die Pascale Odièvre, die französische Klägerin im EGMRVerfahren, stellvertretend formuliert: „Man stellt sich viel vor, aber es ist vor allem eine große Leere. Da ist nichts. Es gibt kein Gesicht, keinen Namen, gar nichts. Und es gibt nicht einmal einen entfernten Verwandten, der Dir Deine Geschichte erzählen kann. Man ist in ein Nichts geboren."95 Angebote, Kinder anonym abzugeben, entwerten zudem die vorhandenen Hilfskonzepte und die regulären Verfahren der Adoptionsvermittlung, die aufgrund ihrer formalen Ausgestaltung nach wie vor am besten geeignet sind, einen Ausgleich der vielschichtigen Interessen zu gewährleisten. Sie passen damit auch nicht zu den Grundprinzipien des freiheitlichen Sozialstaates, der Hilfe zur Wahrnehmung, nicht aber zur Entledigung von Verantwortung leisten soll. ${ }^{96}$

Bei der Parallelproblematik der anonymen Samenspende hat der Gesetzgeber mittlerweile reagiert. Einrichtungen, die heterologe Inseminationen i. S. v. $§ 8$ d Abs. 2 TPG durchführen, sind nach $\S 15$ Abs. 2 TPG verpflichtet, die Daten des Spenders 30 Jahre lang aufzubewahren. Ausdrücklich heißt es in $\S 14$ Abs. 3 S. 1 TPG, dass die datenschutzrechtlichen Verpflichtungen der Gewebeeinrichtung das Recht des Kindes auf Kenntnis der eigenen Abstammung unberührt lassen. Damit wird auch in Kauf genommen, dass die Bereitschaft der Samenspende durch das Anonymitätsverbot zurückgehen wird.

94 Vgl. zur insoweit schwachen Position der Mutter Schwab (Fn. 36), Rn. 559.

95 Zitat nach I. Wiemann, Babyklappe und anonyme Geburt. Hintergründe - Kritik - Alternativen (www.irmela.wiedmann.de).

96 Katzenmaier (Fn. 16), S. 1139. 
Die anonyme Kindesabgabe ist sogar schon nach geltendem Recht unzulässig; der Handlungsbedarf beschränkt sich daher auf den Gesetzesvollzug. Gesetzentwürfe, die anonyme Kindesabgaben zulassen, sind hingegen verfassungswidrig und verdienen nur eins: ,eine anonyme Bestattung, damit niemand die Chance der Exhumierung erhält." "97

97 S. Willutzki, Babyklappe und anonyme Geburt - gibt es eine familienrechtliche Lösung?, in: Diakonisches Werk Berlin-Brandenburg e. V./Caritasverband für das Erzbistum Berlin e. V. (Hg.): Auf den Prüfstand gestellt. Babyklappe und anonyme Geburt, Berlin 2003, S. 34. 